\title{
Leaf Identification Using Fourier Descriptors and Other Shape Features
}

\author{
Abdul Kadir \\ Faculty of Engineering Technology, Universiti Teknikal Malaysia Melaka (UTeM), Malaysia \\ e-mail: abdulkadir@utem.edu.my
}

\begin{abstract}
Leaf identification is a challenging research. So far, many approaches have been proposed. In this paper, an approach that combines Fourier descriptors with other shape features was investigated to identify 100 hundred kinds of leaves. The result shows that the combination of Fourier descriptors and several other shape features can be used to identify leaves with the accuracy rate of $88 \%$. This result indicates that this approach is a promising way for identifying leaves.
\end{abstract}

Keywords: Fourier descriptor, leaf identification, shape features

\section{Introduction}

So far, many researchers have proposed various approaches in identifying leaves in order to develop a system that can help ordinary people to identify plants based on a leaf. The features used in the identification systems can be classified into shape or morphological features, texture features, margin features, vein features and color features. Some researchers combined some of those feature types. For example, Herdiyeni et al. 3] used shape/morphological, texture, and texture features for identifying 30 species of medicinal plants. Kadir et al. [4] applied shape, texture, vein, and color features in identifying 50 kinds of plants. Mallah et al. [7] integrated the shape, texture and margin features in classifying 100 kinds of plants.

Various shape features were used in the leaf identification systems. Hu geometric moments and Zernike orthogonal moments were used by Wang et al. 12. Hu geometric moments were also used by Zulkifli [14. Kulkarni [5] combined features extracted by using pseudo Zernike moments and other features. Meanwhile, aspect ratio, compactness, centroid and vertical and horizontal projections were used by Lee and Chen [6]. However, combining shape features (and other non-shape features) is a challenge because fusion of features does not always give a good accuracy rate.

Various classifiers were involved in the leaf identification systems. Probabilistic neural network (PNN) was used in [3. General Regression Neural Network (GRNN) was applied in [14] and Radial Basis Probabilistic Neural Networks (RBPNN) was tested in [5]. Meanwhile, Linear Discriminant Analysis (LDA) was used by Shabanzade et al. [11. Each classifier has the advantages and disadvantages. For example, PNN is fast to identify a leaf, but it needs a lot of training samples. So, the selection of a classifier is also a challenge. 
The purpose of this research was to explore Fourier descriptors and other shape/morphological features to capture the shape of leaves so it could be used to identify a leaf with high accuracy without any other information such as its color, vein and texture. Other information would be used to increase the higher accuracy in other researches. The Fourier descriptors were chosen based on their good performance in several applications: protein classification [1], vehicular shape-based objects [13] and fingerprint classification [9]. However, some other shape/morphology features were explored to support Fourier descriptors in order to get high accuracy in identifying leaves.

\section{Materials and Methods}

\subsection{Fourier Descriptors}

Fourier descriptors (FD) are usually used for representing the shape of an object by using Fourier transform. Those descriptors have been introduced by Cosgriff in 1960 [8]. The descriptors are calculated based on the contour of the object. If $\mathbf{x}$ is the vector of all $x$ values in the boundary object containing $N$ pixels and $\mathbf{y}$ is the vector of all $y$ values in the boundary object containing $N$ pixels, a complex number of pair of $x$ and $y$ can be written as follows:

$$
\mathbf{z}=\mathbf{x}+j \mathbf{y}
$$

Then, the Fourier descriptors of $z$ can be calculated by using the following formula:

$$
Z[k]=\frac{1}{N} \sum_{m=0}^{N-1} z[m] e^{-j 2 \pi m k / N}, \quad(k=0, \ldots, N-1)
$$

In practice, Fast Fourier Transform (FFT) is used for calculating $Z[k]$.

Some properties in the Fourier descriptors are as follows:

- $Z^{\prime}[k]=S Z[k]$ for scaling;

- $Z^{\prime}[k]=Z[k]+z_{0} \delta[k]$ for translation (So, it influences the DC component Z[0] of the FD);

- $Z^{\prime}[k]=Z[k] e^{j \phi}$ for rotation;

- $Z^{\prime}[k]=Z[k] e^{-j 2 \pi m k / N \phi}$ for shifting the starting point in the boundary from 0 to $m$.

To normalize the Fourier descriptors (FD) from all those properties, some actions are done by using the following pseudo-code:

$(1) G \leftarrow F D$

$(2) G \leftarrow G /|F D(0)|$

$(3) G \leftarrow|G|$

In this case, the step 2 is used for normalizing the descriptors from scaling, translation and moving the starting point, by dividing with the DC component. Meanwhile, the step 3 is used for normalizing the descriptor from rotation by taking the magnitude of the complex numbers. 


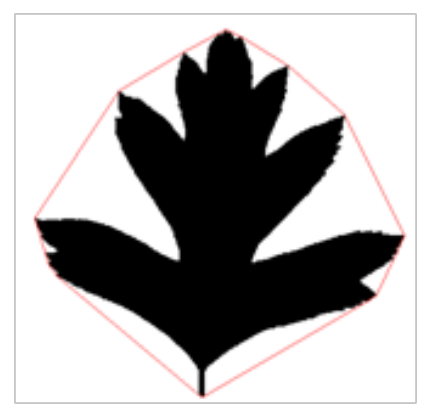

Figure 1. A leaf and its convex hull.

\subsection{Other Shape/Morphology Features}

Other shape/morphology features that can be used in leaf identification systems are aspect factor, roundness factor, irregularity factor [8], solidity [10] and convexity [10. How to calculate all these features is shown in Eq.(1)-(7). Two of them involve convex hull of the leaf. Figure 1 shows an example of leaf convex hull.

$$
\begin{gathered}
\text { aspect ratio }=\frac{\text { width of leaf }}{\text { length of leaf }} \\
\text { roundness factor }=\frac{4 \pi A}{p^{2}} \text {, where } A=\text { leaf area and } p=\text { leaf perimeter } \\
\text { irregularity }=\frac{\text { radius of the maximum circle enclosing the leaf }}{\text { radius of the minimum circle can be contained in the leaf }} \\
\text { solidity }=\frac{\text { area of the leaf }}{\text { area of the convex hull }} \\
\text { convexity }=\frac{\text { perimeter of the leaf convex hull }}{\text { leaf perimeter }}
\end{gathered}
$$

\subsection{Bayes Classifier}

Bayes Classifier follows Bayes rule as shown in Eq.(8] [2].

$$
P\left(\omega_{i} \mid x\right)=\frac{p\left(x \mid \omega_{i}\right) P\left(\omega_{i}\right)}{p(x)}, p(x)=\sum_{i=1}^{c} p\left(x \mid \omega_{i}\right) P\left(\omega_{i}\right)
$$

In the above formula, $p(x)$ is a priori probability of class $\omega_{i} ; P\left(\omega_{i} \mid x\right)$ is a posteriori probability density function (pdf) of $x_{i} ; p\left(x \mid \omega_{i}\right)$ is the class conditional pdf of $x$ given $\omega_{i}$ where $i=1,2, \ldots, c$ and $c$ is number of classes. The data in each class is distributed to the Gaussian distribution $N\left(m_{i}, S_{i}\right)$, where $m_{i}$ is the mean of the class $\omega_{i}$ and $S_{i}$ is the covariance matrix of the class $\omega_{i}$. Then, $x$ is assigned to the class $\omega_{i}$ if it fulfills Eq.99.

$$
P\left(\omega_{i} \mid x\right)>P\left(\omega_{j} \mid x\right), \forall j \neq i
$$




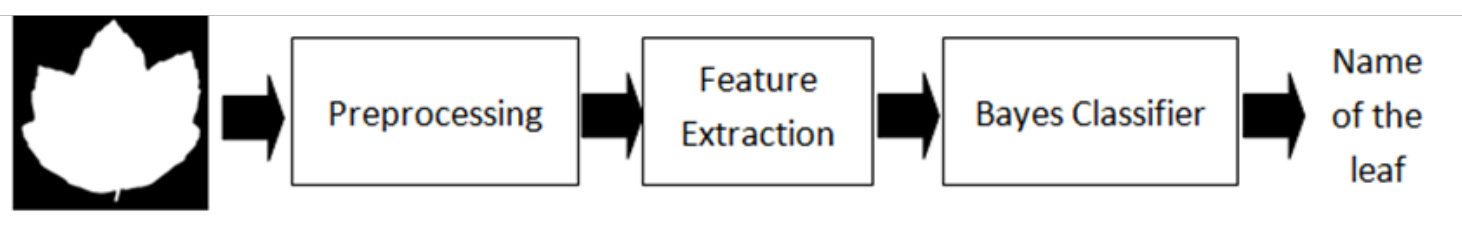

Figure 2. Identification process of a leaf.

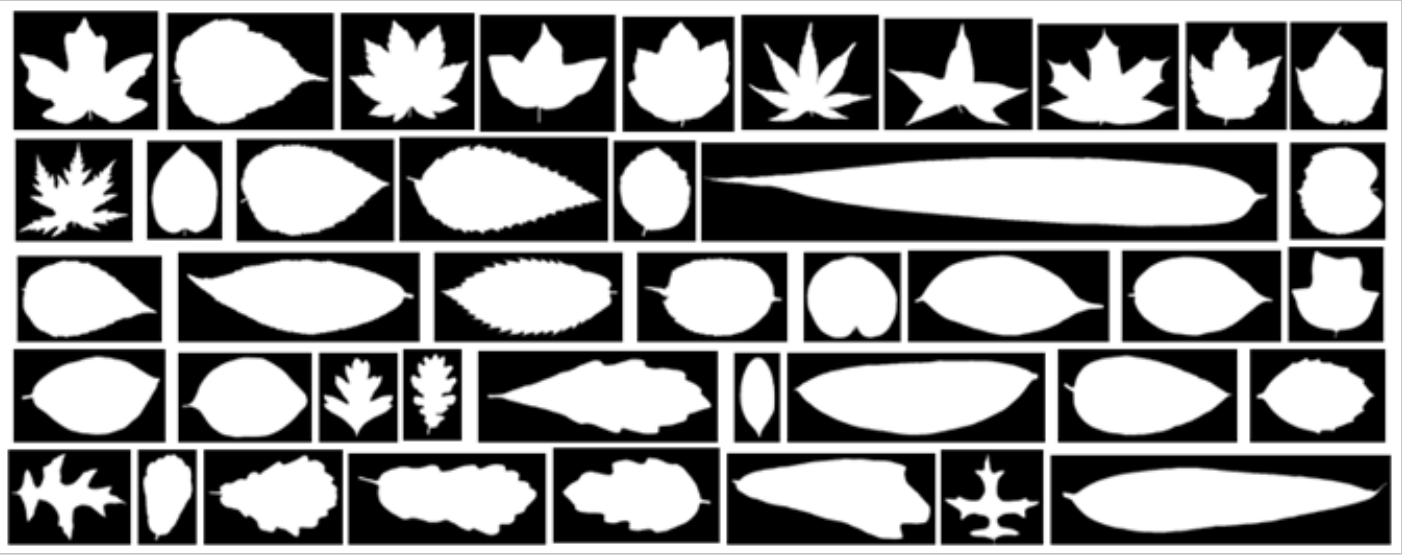

Figure 3. Samples of the dataset.

\section{Proposed System}

The block diagram of the proposed system is illustrated in Fig.(2). The image of leaf was preprocessed so the part of the leaf can be separated from its background by using Canny operator. After that, features of the leaf were extracted. Then, the features were processed by using the Bayes classifiers. In this case, some precalculated features of some leaves were used for decision making in identifying the leaf.

\section{Experiment Results}

To test the system, a dataset containing 100 plants [7] was used. Some of them are shown in Fig.(3). The dataset contains 16 samples of each leaf. $20 \%$ of the samples was used as testing leaves and the rest (80\%) was used as reference leaves. So far, the dataset contains leaves with black and white colors. Therefore, only shape/morphology and margin features can be calculated. In this case, only shape/morphology features were tested.

The accuracy rate was calculated as follows:

$$
\text { Performance }=\frac{\text { Number of relevant images }}{\text { Total number of query }}
$$

Testing based on the above formula shows that the system can gave $88.03 \%$ of accuracy rate.

\section{Conclusion}

The proposed leaf identification system gave $88.03 \%$ of accuracy rate. Actually, this accuracy is lower than the original result by Mallah et al. [7], that included margin and texture features. However, this result 
is good enough in capturing the shape of leaves. So, by adding the margin features and other features, it is possible to increase the accuracy of the system. Therefore, some experiments will be conducted for improving the performance.

\section{References}

[1] A.F. Ali and D.M. Shawky. A novel approach for protein classification using Fourier transfer. World Academy of Science, Engineering and Technology, 4:193-197, 2010.

[2] R.O. Duda, P.E. Hart, and D.G. Stork. Pattern classification. John Wiley \& Sons, 2001. New York.

[3] Y. Herdiyeni, E. Nurfadhilah, E.A.M. Zuhud, E.K. Damayanti, K. Arai, and H. Okumura. A computer aided system for tropical leaf medicinal plant identification. International Journal on Advanced Science, Engneering and Information Technology, 3(1):23-28, 2013.

[4] A. Kadir, L.E. Nugroho, A. Susanto, and P.I. Santosa. Foliage plant retrieval using polar Fourier transform, color moments and vein features. Signal $\&$ Image Processing: An International Journal, $2(3): 1-13,2011$.

[5] A. H. Kulkarni, H.M. Rai, K.A. Jahagirdar, and R.J. Kadkol. A leaf recognition system for classifying plants using RBPNN and pseudo Zernike moments. International Journal of Latest Trends in Engineering and Technology, 2(1):6-11, 2013.

[6] C.L. Lee and S.Y. Chen. Classification for leaf images. In 16th IPPR Conference on Computer Vision, Graphics and Image Processing, volume 8, pages 17-19, 2003.

[7] C. Mallah, J. Cope, and J. Orwell. Plant leaf classification using probabilistic integration of shape, texture and margin features. In Signal Processing, Pattern Recognition and Applications (SPPRA), 2013.

[8] M.S. Nixon and A.S. Aguado. Feature extraction and image processing. Newnes, 2002.

[9] C.H. Park and H. Park. Fingerprint classification using fast Fourier transforms and non linear discriminant analysis. Pattern Recognition, 38(4):495-503, 2005.

[10] J.C. Russ. The Image processing handbook. CRC Press, Boca Raton, 2011.

[11] M. Shabanzade, M. Zahedi, and S. A. Aghvami. Combination of local descriptors and global features for leaf classification. Signal Image Processing: An International Journal, 2(3):23-31, 2011.

[12] X.F. Wang, D.S. Huang, J.X. Du, H. Xu, and L. Heutte. Classification of plant leaf images with complicated background. Applied Mathematics and Computation, 205:916-926, 2008.

[13] R.B. Yadav, N.K. Nishchal, A.K. Gupta, and V.K. Rastogi. Vehicular shape-based objects classification using Fourier descriptors technique. Journal of Scientific \& Industry Research, 68:484-495, 2009.

[14] Z. Zulkifli. Plant leaf identification using moment invariants \& general regression neural network. Master's thesis, Universiti Teknologi Malaysia, 2009. 